\title{
Finding Cliques in Social Networks: A New Distribution-Free Model
}

\author{
Jacob Fox ${ }^{1}$ \\ Department of Mathematics, Stanford University, Stanford, CA 94305, USA \\ jacobfox@stanford.edu
}

Tim Roughgarden ${ }^{2}$

Department of Computer Science, Stanford University, Stanford, CA 94305, USA

\section{Seshadhri}

Department of Computer Science, University of California, Santa Cruz, CA 95064, USA

\section{Fan Wei}

Department of Mathematics, Stanford University, Stanford, CA 94305, USA

\section{Nicole Wein ${ }^{3}$}

EECS, Massachusetts Institute of Technology, Cambridge, MA 02139, USA

\begin{abstract}
We propose a new distribution-free model of social networks. Our definitions are motivated by one of the most universal signatures of social networks, triadic closure - the property that pairs of vertices with common neighbors tend to be adjacent. Our most basic definition is that of a $c$-closed graph, where for every pair of vertices $u, v$ with at least $c$ common neighbors, $u$ and $v$ are adjacent. We study the classic problem of enumerating all maximal cliques, an important task in social network analysis. We prove that this problem is fixed-parameter tractable with respect to $c$ on $c$-closed graphs. Our results carry over to weakly c-closed graphs, which only require a vertex deletion ordering that avoids pairs of non-adjacent vertices with $c$ common neighbors. Numerical experiments show that well-studied social networks tend to be weakly $c$-closed for modest values of $c$.
\end{abstract}

2012 ACM Subject Classification Theory of computation $\rightarrow$ Fixed parameter tractability

Keywords and phrases Graph algorithms, social networks, fixed-parameter tractability

Digital Object Identifier 10.4230/LIPIcs.ICALP.2018.55

Related Version A full version of the paper is available at [29], https://arxiv.org/abs/1804. 07431.

Acknowledgements We would like to thank Christina Gilbert for writing the code to calculate the $c$-closure and weak $c$-closure of networks in the SNAP data sets. We would also like to thank Virginia Vassilevska Williams and Josh Alman for useful conversations about turning our bound into an algorithm.

1 Supported by a Packard Fellowship, an NSF Career Award DMS-1352121, and an Alfred P. Sloan Fellowship

2 Supported by NSF award CCF-1524062

3 Supported by an NSF Graduate Fellowship

(c) (i) acob Fox, Tim Roughgarden, C. Seshadhri, Fan Wei, and Nicole Wein; cc. licensed under Creative Commons License CC-BY

45th International Colloquium on Automata, Languages, and Programming (ICALP 2018). Editors: Ioannis Chatzigiannakis, Christos Kaklamanis, Dániel Marx, and Donald Sannella; Article No. 55; pp. 55:1-55:15 


\section{Introduction}

There has been an enormous amount of important work over the past 15 years on models for capturing the special structure of social networks. This literature is almost entirely driven by the quest for generative (i.e., probabilistic) models. Well-known examples of such models include preferential attachment [6], the copying model [38], Kronecker graphs [13, 39], and the Chung-Lu random graph model $[14,15]$. There is little consensus about which generative model is the "right" one. For example, already in 2006, the survey by Chakrabarti and Faloutsos [12] compares 23 different probabilistic models of social networks, and multiple new such models are proposed every year.

Generative models articulate a hypothesis about what "real-world" social networks look like, how they are created, and how they will evolve in the future. They are directly useful for generating synthetic data and can also be used as a proxy to study the effect of random processes on a network $[3,41,43]$. However, the plethora of models presents a quandary for the design of algorithms for social networks with rigorous guarantees: which of these models should one tailor an algorithm to? One idea is to seek algorithms that are tailored to none of them, and to instead assume only determinstic combinatorial conditions that share the spirit of the prevailing generative models. This is the approach taken in this paper.

There is empirical evidence that many NP-hard optimization problems are often easier to solve in social networks than in worst-case graphs. For example, lightweight heuristics are unreasonably effective in practice for finding the maximum clique of a social network [52]. Similar success stories have been repeatedly reported for the problem of recovering dense subgraphs or communities in social networks $[60,54,42,59]$. To define our notion of "socialnetwork-like" graphs, we turn to one of the most agreed upon properties of social networks triadic closure, the property that when two members of a social network have a friend in common, they are likely to be friends themselves.

\subsection{Properties of social networks}

There is wide consensus that social networks have relatively predictable structure and features, and accordingly are not well modeled by arbitrary graphs. From a structural viewpoint, the most well studied and empirically validated statistical properties of social networks include heavy-tailed degree distributions [6, 11, 23], a high density of triangles [64, 53, 63] and other dense subgraphs or "communities" [26, 32, 46, 47, 40], low diameter and the small world property $[35,36,37,45]$, and triadic closure $[53,63,56]$.

For the problem of finding cliques in networks, it does not help to assume that the graph has small diameter (every network can be rendered small-diameter by adding one extra vertex connected to all other vertices). Similarly, merely assuming a power-law degree distribution does not seem to make the clique problem easier [24]. On the other hand, as we show, the clique problem is tractable on graphs with strong triadic closure properties.

\subsection{Our model: $c$-closed graphs}

Motivated by the empirical evidence for triadic closure in social networks, we define the class of $c$-closed graphs. Figure 1 shows the triadic closure of the network of email communications at Enron [1] and other social networks have been shown to behave similarly [8]. In particular, the more common neighbors two vertices have, the more likely they are to be adjacent to each other. The definition of $c$-closed graphs is a coarse version of this property: we assert that every pair of vertices with $c$ or more common neighbors must be adjacent to each other. 


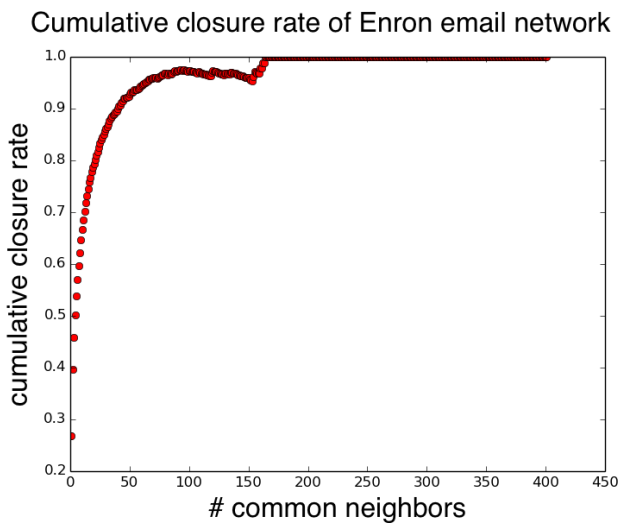

Figure 1 Triadic closure properties of the Enron email graph (36K nodes and 183K edges). Nodes of this network are Enron employees, and there is an edge connecting two employees if one sent at least one email to the other. Given an $x$ value, the $y$-axis shows the cumulative closure rate: the fraction of pairs of nodes with at least $x$ common neighbors that are themselves connected by an edge.

- Definition 1 (c-closed graph). For a positive integer $c$, an undirected graph $G=(V, E)$ is $c$-closed if, whenever two distinct vertices $u, v \in V$ have at least $c$ common neighbors, $(u, v)$ is an edge of $G$.

The parameter $c$ interpolates between a disjoint unions of cliques (when $c=1$ ) and all graphs (when $c=|V|-1$ ). The class of 2-closed graphs is already non-trivial. These are exactly the graphs that do not contain $K_{2,2}$ or a diamond $\left(K_{4}\right.$ minus an edge) as an induced subgraph. For example, graphs with girth at least 5, e.g. constant-degree expanders, are 2-closed. For every $c$, membership in the class of $c$-closed graphs can be checked by squaring the adjacency matrix in $O\left(n^{\omega}\right)$ time, where $\omega<2.373$ is the matrix multiplication exponent.

While the definition of $c$-closed captures important aspects of triadic closure, it is fragile in the sense that a single pair of non-adjacent vertices with many common neighbors prevents the graph from being $c$-closed for a low value of $c$. To address this, we define the more robust notion of weakly c-closed graphs and show that our results carry over to these graphs. Well-studied social networks with thousands of vertices are typically weakly $c$-closed for modest values of $c$ (see the full version [29] for experimental results).

- Definition 2. Given a graph and a value of $c$, a bad pair is a non-adjacent pair of vertices with at least $c$ common neighbors.

Definition 3 (Weakly c-closed graph). A graph is weakly c-closed if there exists an ordering of the vertices $\left\{v_{1}, v_{2}, \ldots, v_{n}\right\}$ such that for all $i, v_{i}$ is in no bad pairs in the graph induced by $\left\{v_{i}, v_{i+1}, \ldots, v_{n}\right\}$.

A graph can be $c$-closed only for large $c$ but weakly $c$-closed for much smaller $c$. Consider the graph $G$ that is a clique of size $k$ with one edge $(u, v)$ missing. $G$ is not $c$-closed for any $c<k-2$. The only bad pair in $G$ is $(u, v)$. The vertex ordering that places $u$ and $v$ at the end demonstrates that $G$ is weakly 1 -closed. Also, the properties of being $c$-closed and weakly $c$-closed are hereditary, meaning that they are closed under taking induced subgraphs. We will use this basic fact often. 


\subsection{Our contributions}

One can study a number of computational problems on $c$-closed (and weakly c-closed) graphs. We focus on the problem of enumerating all maximal cliques, an important problem in social network analysis $[16,58,18,21,57]$. We study fixed-parameter tractability ${ }^{4}$ with respect to $c$. There is a rich literature on fixed-parameter tractability for other graph parameters including treewidth, arboricity, and the size of the output [17].

In a graph $G$, a clique is a subgraph of $G$ in which there is an edge between every pair of vertices. A maximal clique in $G$ is a clique that cannot be made any larger by the addition of some other vertex in $G$. In any graph, all maximal cliques can be listed in $O(m n)$ time per maximal clique [61]. We focus on the following two problems:

1. determining the maximum possible number of maximal cliques in a $c$-closed graph on $n$ vertices.

2. finding algorithms to enumerate all maximal cliques in $c$-closed graphs (that run faster than $O(m n)$ time per maximal clique).

Our main result is that for constant $c$ the number of maximal cliques in a $c$-closed graph on $n$ vertices is $O\left(n^{2-2^{1-c}}\right)$. More specifically, we prove the following bound.

- Theorem 4. Any c-closed graph on $n$ vertices has at most $\min \left\{3^{(c-1) / 3} n^{2}, 4^{(c+4)(c-1) / 2} n^{2-2^{1-c}}\right\}$ maximal cliques.

For example, 3-closed, 4-closed, and 5-closed graphs have $O\left(n^{3 / 2}\right), O\left(n^{7 / 4}\right)$, and $O\left(n^{15 / 8}\right)$ maximal cliques respectively.

The proof of the first bound listed in Theorem 4 extends to weakly $c$-closed graphs, giving the following result.

- Theorem 5. Any weakly c-closed graph on $n$ vertices has at most $3^{(c-1) / 3} n^{2}$ maximal cliques.

See the full version [29] for experimental results showing that well-studied social networks are weakly $c$-closed for modest values of $c$. Note that Theorem 5 is exponential in the even smaller value of $(c-1) / 3$.

Since in any graph all maximal cliques can be listed in $O(m n)$ time per maximal clique, Theorem 4 proves that listing all maximal cliques in a $c$-closed graph is fixed-parameter tractable (i.e. has running time $f(c) n^{\alpha}$ for constant $\alpha$ ). We give an algorithm for listing all maximal cliques in a $c$-closed graph that runs faster than applying the $O(m n)$-per-clique algorithm as a black box. Our algorithm follows naturally from the proof of Theorem 4 and gives the following theorem, where $p(n, c)$ denotes the time to list all wedges (induced 2-paths) in a $c$-closed graph on $n$ vertices. A result of Gąsieniec, Kowaluk, and Lingas [31] implies that $p(n, c)=O\left(n^{2+o(1)} c+c^{(3-\omega-\alpha) /(1-\alpha)} n^{\omega}+n^{\omega} \log n\right)$ where $\omega$ is the matrix multiplication exponent and $\alpha>0.29$.

- Theorem 6. In any c-closed graph, a set of cliques containing all maximal cliques can be generated in time $O\left(p(n, c)+3^{c / 3} n^{2}\right)$. The exact set of all maximal cliques in any c-closed graph can be generated in time $O\left(p(n, c)+3^{c / 3} 2^{c} c n^{2}\right)$.

Non-trivial lower bounds for the number of maximal cliques in a $c$-closed graph were previously known only for extreme values of $c$. A 2-closed graph can have $n^{3 / 2}$ maximal

4 A problem is said to be fixed-parameter tractable with respect to a parameter $k$ if there is an algorithm that solves it in time at most $f(k) n^{\alpha}$ where $f$ can be an arbitrary function but $\alpha$ is a constant. 
cliques [22]. The classic Moon-Moser graph (with additional isolated vertices) is $(n-2)$-closed and has $3^{\lfloor n / 3\rfloor}$ maximal cliques [44]. This graph consists of the complete multipartite graph with $\lfloor n / 3\rfloor$ parts of size 3 , and possibly additional isolated vertices. By taking a disjoint union of $n /(c+2)$ Moon-Moser graphs on $(c+2)$ vertices, we can construct a $c$-closed graph on $n$ vertices with $\Omega\left(c^{-1} 3^{c / 3} n\right)$ maximal cliques for all $n \geq c$. We give improved lower bounds for intermediate values of $c$.

- Theorem 7. For any positive integer $c$, there are c-closed graphs with $n$ vertices and $\Omega\left(c^{-3 / 2} 2^{c / 2} n^{3 / 2}\right)$ maximal cliques.

It is an open problem to determine the exact exponent of $n$ (between $3 / 2$ and $2-2^{1-c}$ ) in the expression for the maximum number of maximal cliques in a $c$-closed graph.

\subsection{Related work}

There are only a few algorithmic results for graph classes motivated by social networks. Although a number of NP-hard problems remain NP-hard on graphs with a power-law degree distribution [25], several problems in $\mathrm{P}$ have been shown to be easier on such graphs. Brach, Cygan, Lacki, and Sankowski [10] give faster algorithms for transitive closure, maximum matching, determinant, PageRank and matrix inverse. Borassi, Crescenzi, and Trevisan [9] assume several axioms satisfied by real-world graphs, one being a power-law degree distribution, and give faster algorithms for diameter, radius, distance oracles, and computing the most "central" vertices. Motivated by triadic closure, Gupta, Roughgarden, and Seshadhri [33] define triangle-dense graphs and prove relevant structural results. Intuitively, they prove that if a constant fraction of two-hop paths are closed into triangles, then the graph must contain many dense clusters.

For general graphs, Moon and Moser prove that the maximum possible number of maximal cliques in a graph on $n$ vertices is $3^{n / 3}$ (realized by a complete $n / 3$-partite graph) [44]. Tomita, Tanaka, and Takahashi prove that the time to generate all maximal cliques in any $n$-vertex graph is also $O\left(3^{n / 3}\right)[58]$.

The clique problem has been studied on 2-closed graphs (under a different name). Eschen, Hoang, Spinrad, and Sritharan [22] show that the maximum number of maximal cliques in a 2-closed graph is $O\left(n^{3 / 2}\right)$. They also show a matching lower bound via a projective planes construction. Suppose $n=p^{2}+p+1$ for a positive integer $p$ and consider a finite projective plane on $n$ points (and hence with $n$ lines, see e.g. [2]). Let $G$ denote the bipartite graph representing the point-line incidence matrix. The defining properties of finite projective planes imply that no two vertices have two common neighbors, so the 2-closed condition is vacuously satisfied. Every vertex of $G$ has degree $p+1$, so the graph has $\Theta\left(n^{3 / 2}\right)$ edges, each a maximal clique.

The clique problem has also been studied on other special classes of graphs such as graphs embeddable on a surface [19] and graphs of bounded degeneracy [20]. Degeneracy is a measure of everywhere sparsity. More formally, the degeneracy of a graph $G$ is the smallest value $d$ such that every nonempty subgraph of $G$ contains a vertex of degree at most $d$. Eppstein et al. show that the maximum number of maximal cliques in a graph of degeneracy $d$ is $O\left(n 3^{d / 3}\right)$. The degeneracy of a graph, however, can be much larger than its $c$-closure. For example, the degeneracy of a graph is at least the size of a maximum clique, while even in 1-closed graphs, the size of the maximum clique can be arbitrarily large.

Clique counting is a classical problem in extremal combinatorics. One fundamental question is to count the minimum number of cliques in graphs with fixed number of edges i.e. to show that graphs with few cliques must have few edges. This simple question turns 
out to be a complex problem, and is settled for $K_{3}$ by Razborov [50] by flag algebra, $K_{4}$ by Nikiforov [48] by a combination of combinatorics and analytical arguments, and all $K_{t}$ by Reiher [51] by generalizing the argument of flag algebra to all sizes of cliques.

There has also been a long line of work in combinatorics on counting (not necessarily maximal) cliques in graphs with certain excluded subgraphs, subdivisions, or minors. Most recently, Fox and Wei give an asymptotically tight bound on the maximum number of cliques in graphs with forbidden minors [28], and an upper bound on the maximum number of cliques in graphs with forbidden subdivisions or immersions [27].

Many problems in combinatorics can be phrased as counting the number of cliques or independent sets in a (hyper)graph. For example, the problems of finding the volume of the metric polytope and counting the number of $n$-vertex $H$-free graphs (for any fixed subgraph $H$ ) can be translated into clique counting problems. The recently developed "container method" $[5,55]$ is a powerful tool to bound the number of cliques in (hyper)graphs and can be used to tackle a great range of problems.

\subsection{Organization}

In Section 2 we prove the first bound listed in Theorem 4, state Theorem 5, and introduce the proof of Theorem 6. In Section 3 we prove the second bound listed in Theorem 4 (which has improved dependence on $n$ ). In Section 4 we prove Theorem 7 .

See the full version [29] for the proof of Theorem 6 .

\subsection{Notation}

All graphs $G(V, E)$ are simple, undirected and unweighted. For any $v \in V$, let $N(v)$ denote the neighborhood of $v$. When the current graph is ambiguous, $N_{G}(v)$ will denote the neighborhood of $v$ in $G$. For any $S \subseteq V$, let $G[S]$ denote the subgraph of $G$ induced by $S$.

\section{Initial Bound and Algorithm}

\subsection{Bound on number of maximal cliques}

In this section, we prove the following bound on the number of maximal cliques in a $c$-closed graph and show that this bound carries over to weakly $c$-closed graphs. Let $F(n, c)$ denote the maximum possible number of maximal cliques in a $c$-closed graph on $n$ vertices. The following theorem uses a natural peeling process and obtain an initial upper bound on the number of maximal cliques. A more involved analysis, Theorem 11 which gives a tighter upper bound, is delayed to later.

- Theorem 8 (restatement of part of Theorem 4). For all positive integers $c, n$, we have $F(n, c) \leq 3^{(c-1) / 3} n^{2}$.

Proof. Let $G$ be a $c$-closed graph on $n$ vertices and let $v \in V(G)$ be an arbitrary vertex. Every maximal clique $K \subseteq G$ is of one of the following types:

1. The clique $K$ does not contain vertex $v$; and $K$ is maximal in $G \backslash\{v\}$.

2. The clique $K$ contains vertex $v$; and $K \backslash\{v\}$ is maximal in $G \backslash\{v\}$.

3. The clique $K$ contains vertex $v$; and $K \backslash\{v\}$ is not maximal in $G \backslash\{v\}$.

Bounding the number of maximal cliques of type 1 and 2 is straightforward because every such clique can be obtained by starting with a clique maximal in $G \backslash\{v\}$ and extending it to include vertex $v$ if possible. Therefore, the number of maximal cliques of types 1 and 2 combined is at most $F(n-1, c)$. 


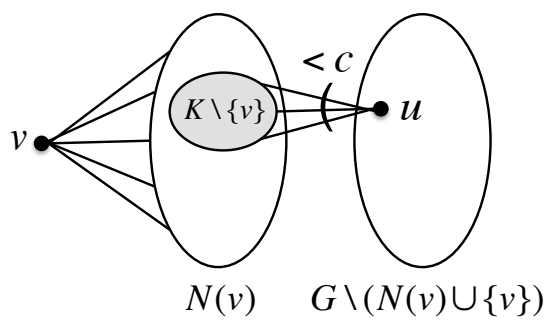

Figure 2 A maximal clique $K$ of type 3: $K$ contains vertex $v$ and $K \backslash\{v\}$ is not maximal in $G \backslash\{v\}$. Property $\mathrm{C}$ asserts that there exists a vertex $u \notin N(v)$ whose neighborhood contains $K \backslash\{v\}$. Since $G$ is $c$-closed, $|N(u) \cap N(v)|<c$.

Type 3 cliques are maximal in $N(v)$, but not in $G \backslash\{v\}$. We will prove that the number of maximal cliques of type 3 is at most $3^{(c-1) / 3} n$, crucially using the $c$-closed property. Figure 2 shows a maximal clique $K$ of type 3 .

We claim that each type 3 maximal clique $K$ satisfies the following three properties.

A) $K \backslash\{v\}$ is a clique in the neighborhood of $v$, and

B) $K \backslash\{v\}$ is not in the neighborhood of any other vertex in $N(v)$.

C) There exists a vertex $u \notin N(v)$ whose neighborhood contains $K \backslash\{v\}$.

Property A is clear since $K$ is a clique containing $v$. Property B is true because if we can extend $K \backslash\{v\}$ to include some vertex $w \in N(v)$, then $K$ can also be extended to include $w$ which contradicts the fact that $K$ is maximal. To see property C, note that since $K \backslash\{v\}$ is not a maximal clique in $G \backslash\{v\}$ we can extend the clique $K \backslash\{v\}$ to include some vertex in $G \backslash\{v\}$. By property B, we can extend $K \backslash\{v\}$ to include some vertex $u$ not in $N(v)$.

Let $u$ be as in property C. Then $K \backslash\{v\}$ must be a maximal clique in $G[N(v) \cap N(u)]$ because otherwise we could extend $K \backslash\{v\}$ to some other vertex in $N(v) \cap N(u)$, which contradicts property B.

Thus, the number of type 3 maximal cliques is at most

$$
\sum_{u \in G \backslash(N(v) \cup\{v\})} F(|N(u) \cap N(v)|, c) .
$$

Since $G$ is $c$-closed, $|N(u) \cap N(v)|<c$ for all vertices $u \notin N(v)$. Then since any $k$-vertex graph has at most $3^{k / 3}$ maximal cliques [44],

$$
F(|N(u) \cap N(v)|, c) \leq 3^{(c-1) / 3} .
$$

Thus, the number of type 3 maximal cliques in $G$ is at most $3^{(c-1) / 3} n$.

Counting all three types of maximal cliques, we have the following recursive inequality:

$$
F(n, c) \leq F(n-1, c)+3^{(c-1) / 3} n .
$$

By induction on $n$ with the base case $F(1, c)=1$, this gives

$$
F(n, c) \leq 3^{(c-1) / 3}\left(\begin{array}{c}
n+1 \\
2
\end{array}\right) \leq 3^{(c-1) / 3} n^{2} .
$$

Note that $v$ was chosen arbitrarily and the proof is valid as long as " $|N(u) \cap N(v)|<c$ for all vertices $u \notin N(v)$ ". Thus, in each recursive level, we only require the existence of a vertex $v$ in no bad pairs. Equivalently, it suffices to have an ordering of the vertices $\left\{v_{1}, v_{2}, \ldots, v_{n}\right\}$ such that for all $i, v_{i}$ is in no bad pairs in the graph induced by $\left\{v_{i}, v_{i+1}, \ldots, v_{n}\right\}$. This is exactly the definition of a weakly $c$-closed graph. Thus, we get the following theorem. 
- Theorem 9 (Restatement of Theorem 5). For any positive integers $c, n$, there are at most $3^{(c-1) / 3} n^{2}$ maximal cliques in an n-vertex weakly c-closed graph.

\subsection{Algorithm to generate all maximal cliques}

Recall that $p(n, c)$ denotes the time to list all wedges (induced 2-paths) in a $c$-closed graph on $n$ vertices. A result of Gasieniec, Kowaluk, and Lingas [31] implies that $p(n, c)=$ $O\left(n^{2+o(1)} c+c^{(3-\omega-\alpha) /(1-\alpha)} n^{\omega}+n^{\omega} \log n\right)$ where $\omega$ is the matrix multiplication exponent and $\alpha>0.29$.

- Theorem 10 (restatement of Theorem 6). A superset of the maximal cliques in any c-closed graph can be generated in time $O\left(p(n, c)+3^{c / 3} n^{2}\right)$. The exact set of all maximal cliques in any c-closed graph can be generated in time $O\left(p(n, c)+3^{c / 3} 2^{c} c n^{2}\right)$.

The algorithm follows naturally from the proof of Theorem 8 with two additional ingredients:

- A preprocessing step to enumerate all wedges in the graph speeds up the later process of finding the intersection of the neighborhoods of two vertices (i.e. $N(u) \cap N(v)$ from the proof of Theorem 8).

- An algorithm of Tomita, Tanaka, and Takahashi [58] generates all maximal cliques in any $n$-vertex graph in time $O\left(3^{n / 3}\right)$. We apply this to the recursive calls on the small induced subgraphs $G[N(u) \cap N(v)]$, which have less than $c$ vertices, that arise in handling the type 3 cliques in the proof of Theorem 8.

We defer the full algorithm description and runtime analysis to the full version [29].

\section{Improved Bound}

Recall that $F(n, c)$ is the maximum number of maximal cliques in a $c$-closed graph on $n$ vertices.

- Theorem 11 (restatement of part of Theorem 4). For all positive integers c,n, we have $F(n, c) \leq 4^{(c+4)(c-1) / 2} n^{2-2^{1-c}}$.

The structure of the proof is similar to that of Theorem 8 . We get an improved bound by a separate analysis depending on whether $G$ has a vertex of "high" degree. This idea appears in the result of Eschen et al. [22], who prove the result for the $c=2$ case.

We will require the following simple lemma.

- Lemma 12. For any $v, G[N(v)]$ is a $(c-1)$-closed graph.

Proof. Consider pair $x, y \in N(v)$ with $c-1$ common neighbors in $G[N(v)]$. Since vertex $v \notin N(v)$ is also a common neighbor of $x$ and $y, x$ and $y$ have $c$ common neighbors in $G$. Thus, $(x, y)$ is an edge.

Proof of Theorem 11. Let $G=(V, E)$ be a $c$-closed graph on $n$ vertices with $F(n, c)$ maximal cliques. Let $\Delta(G)$ be the maximum degree of $G$.

Case 1: $\Delta(G) \leq n^{1 / 2}$.

By Lemma 12 for all $v \in V(G), G[N(v)]$ is $(c-1)$-closed. Then, since the number of maximal cliques containing $v$ is exactly the number of maximal cliques in $G[N(v)]$, we have

$$
F(n, c) \leq n F(\Delta(G), c-1) .
$$


Case 2: $\Delta(G)>n^{1 / 2}$.

Let $v \in V$ be a vertex of degree $\Delta(G)$. We will count the maximal cliques containing at least one vertex in $N(v) \cup\{v\}$, delete $N(v) \cup\{v\}$, and recurse.

Since the number of maximal cliques containing $v$ is exactly the number of maximal cliques in $G[N(v)], v$ is in at most $F(\Delta(G), c-1)$ maximal cliques. It remains to bound the number the maximal cliques that contain some vertex in $N(v)$ but not $v$ itself. Such a clique must contain some vertex in $u \in V \backslash(N(v) \cup\{v\})$ (otherwise, it would not be maximal). Let $\mathcal{K}$ be the set of such cliques. We will bound $|\mathcal{K}|$ by grouping the maximal cliques $K$ in $\mathcal{K}$ based on which vertices of $N(v)$ are in $K$. For nonempty $S \subseteq N(v)$, let $N(S)$ denote $\bigcap_{u \in S} N(u)$. Also, let $N_{2}(v)$ denote the set of vertices of distance exactly 2 from $v$. Let us bound the number of cliques $K \in \mathcal{K}$ such that $K \cap N(v)=S$. The other vertices in $K$ must be in $N(S) \cap N_{2}(v)$. By Lemma 12, $G\left[N(S) \cap N_{2}(v)\right]$ is a $(c-1)$-closed graph. The number of cliques $K \in \mathcal{K}$ such that $K \cap N(v)=S$ is at most $F\left(\left|N(S) \cap N_{2}(v)\right|, c-1\right)$. Summing over all subsets $S \subseteq N(v)$, we have

$$
|\mathcal{K}| \leq \sum_{S \subseteq N(v)} F\left(\left|N(S) \cap N_{2}(v)\right|, c-1\right) .
$$

For all $u \in N_{2}(v)$, since $u$ and $v$ are not adjacent, $|N(u) \cap N(v)|<c$ (because $G$ is $c$-closed). Each vertex in $N_{2}(v)$ can be in $N(S)$ for only $2^{c-1}$ sets $S \subseteq N(v)$, implying

$$
\sum_{S \subseteq N(v)}\left|N(S) \cap N_{2}(v)\right| \leq\left|N_{2}(v)\right| 2^{c-1} \leq \min \left\{(\Delta(G))^{2}, n\right\} 2^{c-1} .
$$

We want to determine for all $S$ the value of $\left|N(S) \cap N_{2}(v)\right|$ that maximizes the upper bound for $|\mathcal{K}|$ in Inequality (2) subject to the constraint in Inequality (3). Later, we prove our bound on $F$ (from the theorem statement) by induction on $n$ and $c$. In fact, we show that $F(n, c)$ is bounded by

$$
F_{0}(n, c)=4^{(c+4)(c-1) / 2} n^{2-2^{1-c}},
$$

the desired upper bound for $F(n, c)$ that we are trying to prove by induction. Since $F_{0}(n, c)$ is convex in $n$, by the inductive hypothesis we can apply Jenson's inequality on Inequality (2). Jenson's inequality implies that the upper bound on $|\mathcal{K}|$ is maximized by setting $\left|N(S) \cap N_{2}(v)\right|$ to be as large as possible (note that it cannot exceed $\Delta(G)$ ) for as many $S$ as possible until the bound in Inequality (3) is met and setting the rest to be 0 . By Inequality (3), the number of non-zero terms $\left|N(S) \cap N_{2}(v)\right|$ we sum over is at most $\Delta(G)^{-1} \min \left\{(\Delta(G))^{2}, n\right\} 2^{c-1} \leq \min \left\{\Delta(G), \frac{n}{\Delta(G)}\right\} 2^{c-1}$. Thus, we have the following continuation of Inequality (2).

$$
|\mathcal{K}| \leq \sum_{S \subseteq N(v)} F\left(\left|N(S) \cap N_{2}(v)\right|, c-1\right) \leq \min \left\{\Delta(G), \frac{n}{\Delta(G)}\right\} 2^{c-1} F_{0}(\Delta(G), c-1) .
$$

Recall $|\mathcal{K}|$ is the number of maximal cliques that contain some vertex in $N(v)$ but not $v$ itself, so we combine Inequality (4) with the observation (from the beginning of case 2 ) that $v$ is in at most $F(\Delta(G), c-1)$ maximal cliques to conclude that the number of maximal cliques containing at least one vertex in $N(v) \cup\{v\}$ is at most

$$
F_{0}(\Delta(G), c-1)\left(1+\min \left\{\Delta(G), \frac{n}{\Delta(G)}\right\} 2^{c-1}\right) \leq F_{0}(\Delta(G), c-1) \min \left\{\Delta(G), \frac{n}{\Delta(G)}\right\} 2^{c} .
$$

Then, recursing on $G \backslash(N(v) \cup\{v\})$, we have:

$$
F(n, c)<F_{0}(\Delta(G), c-1) \min \left\{\Delta(G), \frac{n}{\Delta(G)}\right\} 2^{c}+F(n-\Delta(G), c) .
$$


Combining the low and high degree bounds on $F(n, c)$, we get the following recurrence.

$$
\begin{aligned}
& F(n, 1)=n, \quad F(1, c)=1 \\
& F(n, c) \leq \begin{cases}n F(\Delta(G), c-1) & \Delta(G) \leq n^{1 / 2} \\
F_{0}(\Delta(G), c-1) \frac{n}{\Delta(G)} 2^{c}+F(n-\Delta(G), c) & \Delta(G)>n^{1 / 2}\end{cases}
\end{aligned}
$$

The remainder of the proof shows inductively that the recurrence implies the desired bound $F(n, c) \leq F_{0}(n, c) \leq n^{2-2^{1-c}} 2^{(c+4)(c-1) / 2}$. The desired bound holds in the two base cases. For the inductive case, we need to show that

$$
n^{2-2^{1-c}} 2^{(c+4)(c-1) / 2} \geq \begin{cases}n \Delta(G)^{2-2^{2-c}} 2^{(c+3)(c-2) / 2} & \Delta(G) \leq n^{1 / 2} \\ \Delta(G)^{2-2^{2-c}} 2^{(c+3)(c-2) / 2} \frac{n}{\Delta(G)} 2^{c} & \\ +(n-\Delta(G))^{2-2^{1-c}} 2^{(c+4)(c-1) / 2} & \Delta(G)>n^{1 / 2} .\end{cases}
$$

In the $\Delta(G) \leq n^{1 / 2}$ case, the expression is maximized when $\Delta(G)=n^{1 / 2}$. Thus,

$$
n \Delta(G)^{2-2^{2-c}} 2^{(c+3)(c-3) / 2} \leq n^{1+\frac{1}{2}\left(2-2^{2-c}\right)} 2^{(c+3)(c-2) / 2}<n^{2-2^{1-c}} 2^{(c+4)(c-1) / 2},
$$

as desired.

For the $\Delta(G)>n^{1 / 2}$ case, the second term of the expression can be written as $\left(n\left(1-\frac{\Delta(G)}{n}\right)\right)^{2-2^{1-c}} 2^{(c+4)(c-1) / 2}$. We use the following claim.

- Claim 13. $(1-x)^{k} \leq 1-\frac{x k}{2}$ for any $0<x \leq 1 / 2$ and $1 \leq k \leq 2$

Proof. For any $y \in(0,1),(1-y) \leq e^{-y}$ and $e^{-y} \leq 1-y / 2$. Thus, $(1-x)^{k} \leq e^{-x k} \leq 1-x k / 2$.

Applying the claim with $x=\frac{\Delta(G)}{n}$ and $k=2-2^{1-c}$, it suffices to show that

$n^{2-2^{1-c}} 2^{(c+4)(c-1) / 2} \geq \Delta(G)^{2-2^{2-c}} 2^{(c+3)(c-2) / 2} \frac{n}{\Delta(G)} 2^{c}+n^{2-2^{1-c}}\left(1-\frac{\Delta(G)\left(2-2^{1-c}\right)}{2 n}\right) 2^{(c+4)(c-1) / 2}$.

or equivalently that

$$
\Delta(G)^{2-2^{2-c}} 2^{(c+3)(c-2) / 2} \frac{n}{\Delta(G)} 2^{c}-n^{2-2^{1-c}} \frac{\Delta(G)\left(2-2^{1-c}\right)}{2 n} 2^{(c+4)(c-1) / 2} \leq 0 .
$$

Simplifying the left-hand side of the above inequality and using the fact that $c \geq 1$ :

$$
\begin{aligned}
& \left.\Delta(G)^{2-2^{2-c}} 2^{(c+3)(c-2) / 2} \frac{n}{\Delta(G)} 2^{c}-n^{2-2^{1-c}} \frac{\Delta(G)\left(2-2^{1-c}\right)}{2 n}\right) 2^{(c+4)(c-1) / 2} \\
& =n \Delta(G)^{1-2^{2-c}} 2^{\left(c^{2}+c-6\right) / 2}-n^{1-2^{1-c}} \Delta(G)\left(1-2^{-c}\right) 2^{\left(c^{2}+3 c-4\right) / 2} \\
& \leq 2^{\left(c^{2}+c-6\right) / 2}\left(n \Delta(G)^{1-2^{2-c}}-n^{1-2^{1-c}} \Delta(G)\left(1-2^{-c}\right) 2^{c+1}\right) \\
& =2^{\left(c^{2}+c-6\right) / 2}\left(n \Delta(G)^{1-2^{2-c}}-n^{1-2^{1-c}} \Delta(G)\left(2^{c+1}-2\right)\right) \\
& \leq 2^{\left(c^{2}+c-6\right) / 2}\left(n \Delta(G)^{1-2^{2-c}}-n^{1-2^{1-c}} \Delta(G)\right) \\
& =2^{\left(c^{2}+c-6\right) / 2} n^{1-2^{1-c}} \Delta(G)^{1-2^{2-c}}\left(n^{2^{1-c}}-\Delta(G)^{2^{2-c}}\right) \leq 0 .
\end{aligned}
$$

The last inequality holds because $\Delta \geq n^{1 / 2}$.

Like the proof of the initial bound (Theorem 8), the proof of the improved bound (Theorem 11) also suggests an algorithm for generating the set of maximal cliques involving the preprocessing step of listing the set of all wedges in the graph. However, this algorithm is not asymptotically faster than the algorithm from Theorem 10 since its dependence on $n$ still includes $p(n, c)$ and we omit it. 


\section{$4 \quad$ Lower bound}

- Theorem 14 (restatement of Theorem 7). For any positive integer $c$, we can construct graphs which are $c$-closed and with $\Omega\left(c^{-3 / 2} 2^{c / 2} n^{3 / 2}\right)$ maximal cliques.

\section{Construction}

We suppose that $c$ is even and $n$ is a multiple of $c$. We can do this with only an absolute constant factor loss in the bound, which is allowable. We start with a graph $H$ on $v=2 n / c$ vertices with girth 5 and the maximum possible number of edges, which is $\Omega\left(v^{3 / 2}\right)$ [30].

We construct our $c$-closed graph $G$ on $n$ vertices from $H$ in the following way. For each vertex $x \in V(H)$, we replace it with a vertex set $U_{x}$ with $c / 2$ vertices. Therefore, there are $|V(H)| \cdot c / 2=n$ vertices in $G$. The adjacency relation of $G$ is as follows.

- Add all edges within each $U_{x}$ so that $U_{x}$ is a clique for all $x \in V(H)$.

- For any edge $(x, y)$ of $H$, we place edges between the vertex sets $U_{x}$ and $U_{y}$ such that the bipartite graph between $U_{x}$ and $U_{y}$ consists of a complete bipartite graph minus a perfect matching.

- For any distinct and nonadjacent $x, y \in V(H)$, there are no edges between $U_{x}$ and $U_{y}$.

Theorem 14 follows from the next two claims.

Claim 15. The graph $G$ constructed is c-closed.

Proof. It suffices to check that for any two non-adjacent vertices in $G$, they have at most $c-1$ common neighbors. By the construction, there are only two types of non-adjacent vertices:

Case 1: The non-adjacent pair $u, v \in V(G)$ are such that $u \in U_{x}, v \in U_{y}$ and $x, y \in V(H)$ are disitinct and non-adjacent in $H$.

In this case, there are no edges between $U_{x}, U_{y}$, and the common neighbors of $u, v$ are such that there is a vertex $z \in V(H)$ such that $(x, z),(y, z)$ are both edges in $H$. Since $H$ has girth 5 , there is at most one such $z \in H$ given $x, y$, as otherwise $H$ would contain a $C_{4}$. Vertex $u \in V(G)$ is adjacent to exactly $\left|U_{z}\right|-1=(c / 2)-1$ vertices in $U_{z}$, so $u, v$ can have at most $(c / 2)-1$ common neighbors.

Case 2: The non-adjacent pair $u, v \in V(G)$ is such that $u \in U_{x}, v \in U_{y}$ and $x, y \in V(H)$ are adjacent in $H$.

In this case, $u$ and $v$ are adjacent to all other vertices in $U_{x} \cup U_{y}$, so they have $c-2$ common neighbors in $U_{x} \cup U_{y}$. Suppose for contradiction that $u, v$ have some other common neighbor $w$ and $w \in U_{z}$ for some $z \neq x, y$. This implies that $(w, x),(w, y)$ are both edges in $H$. However, $(x, y)$ is already an edge in $H$ by the assumption of this case. This implies that $H$ contains a triangle, which contradicts the fact that $H$ has girth 5 .

Combining both cases, we know that $G$ is $c$-closed.

- Claim 16. There are $\Omega\left(c^{-3 / 2} 2^{c / 2} n^{3 / 2}\right)$ maximal cliques in $G$.

Proof. For any edge $(x, y)$ of $H$, picking one endpoint of each non-edge in $U_{x} \cup U_{y}$ gives a maximal clique. Thus for each edge $(x, y)$ of $H$, there are exactly $2^{\left|U_{x}\right|}=2^{c / 2}$ maximal cliques.

There are $\Omega\left(|V(H)|^{3 / 2}\right)=\Omega\left((2 n / c)^{3 / 2}\right)$ edges in $H$. As each of the maximal cliques obtained are distinct, we obtain $\Omega\left(2^{c / 2} \cdot(2 n / c)^{3 / 2}\right)=\Omega\left(c^{-3 / 2} 2^{c / 2} n^{3 / 2}\right)$ maximal cliques. 


\section{Open problems and future directions}

\section{Direct improvement of our results}

- Determine the exact dependence on $n$ for the maximum possible number of of maximal cliques in a $c$-closed graph. We have proven (up to constant dependence on $c$ ) that this number is between $n^{3 / 2}$ and $n^{2-2^{1-c}}$.

- Find a faster algorithm for listing the set of all wedges (induced 2-paths) in a c-closed graph (this would improve the runtime of Algorithm from Theorem 10).

\section{Further exploration of c-closed graphs}

- Study the densest $k$-subgraph problem, a generalization of the clique problem, on $c$-closed graphs. The input to the problem is a graph $G$ and a parameter $k$, and the goal is to to find the subgraph of $G$ on $k$ vertices with the most edges. Unlike the clique problem, densest $k$-subgraph is NP-hard even for 2-closed graphs (more specifically, for graphs of girth 6) [49]. For general graphs, the best-known approximation algorithm has approximation ratio roughly $O\left(n^{1 / 4}\right)[7]$ and under certain average-case hardness assumptions (concerning the planted clique problem), constant-factor approximation algorithms do not exist [4].

- Determine which other NP-hard problems are fixed-parameter tractable with respect to $c$.

- Determine which problems in P have faster algorithms on $c$-closed graphs.

\section{Other model-free definitions of social networks}

- Explore other graph classes motivated by the well-established signatures of social networks (described in the introduction): heavy-tailed degree distributions, high triangle density, dense "communities", low diameter and the small world property, and triadic closure.

- Determine other model-free definitions of social networks, for example, those motivated by 4-vertex subgraph frequencies. Ugander et al. [62] and subsequently Seshadhri [34] computed 4-vertex subgraph counts in a variety of social networks and the frequencies observed are far different than what one would expect from a random graph. In particular, social networks tend to have far fewer induced 4-cycles than random graphs.

\section{References}

1 Enron email dataset. URL: https://www.cs.cmu.edu/ ./enron/.

2 A. Abraham and R. Sandler. An Introduction to Finite Projective Planes. Dover, 2015.

3 R. Albert, H. Jeong, and A.-L. Barabási. Error and attack tolerance of complex networks. Nature, 406:378-382, 2000.

4 N. Alon, S. Arora, R. Manokaran, D. Moshkovitz, and O. Weinstein. Inapproximability of densest $\kappa$-subgraph from average case hardness. Unpublished manuscript, 2011.

5 J. Balogh, R. Morris, and W. Samotij. Independent sets in hypergraphs. J. Amer. Math. Soc., 28(3):669-709, 2015.

6 A.-L. Barabasi and R. Albert. Emergence of scaling in random networks. Science, 286:509512, 1999. doi:10.1126/science.286.5439.509.

7 A. Bhaskara, M. Charikar, E. Chlamtac, U. Feige, and A. Vijayaraghavan. Detecting high log-densities: an $O\left(n^{1 / 4}\right)$ approximation for densest $k$-subgraph. In Proceedings of the 2010 ACM Symposium on Theory of Computing, pages 201-210. ACM, 2010.

8 M. Bloznelis and V. Kurauskas. Clustering function: a measure of social influence. CoRR, abs/1207.4941, 2012. URL: http://arxiv.org/abs/1207.4941, arXiv:1207.4941. 
9 M. Borassi, P. Crescenzi, and L. Trevisan. An axiomatic and an average-case analysis of algorithms and heuristics for metric properties of graphs. arXiv preprint arXiv:1604.01445, 2016.

10 P. Brach, M. Cygan, J. Łącki, and P. Sankowski. Algorithmic complexity of power law networks. In Proceedings of the Twenty-Seventh Annual ACM-SIAM Symposium on Discrete Algorithms, pages 1306-1325. SIAM, 2016.

11 A. Broder, R. Kumar, F. Maghoul, P. Raghavan, S. Rajagopalan, R. Stata, A. Tomkins, and J. Wiener. Graph structure in the web. Computer Networks, 33:309-320, 2000.

12 D. Chakrabarti and C. Faloutsos. Graph mining: Laws, generators, and algorithms. ACM Computing Surveys, 38(1), 2006. doi:10.1145/1132952.1132954.

13 D. Chakrabarti, Y. Zhan, and C. Faloutsos. R-MAT: A recursive model for graph mining. In SIAM Conference on Data Mining, pages 442-446, 2004. URL: http://siam.org/ proceedings/datamining/2004/dm04_043chakrabartid.pdf.

14 F. Chung and L. Lu. The average distances in random graphs with given expected degrees. Proc. Natl. Acad. Sci. USA, 99(25):15879-15882, 2002. doi:10.1073/pnas. 252631999.

15 F. Chung and L. Lu. Connected components in random graphs with given degree sequences. Ann. Comb., 6:125-145, 2002. doi:10.1007/PL00012580.

16 A. Conte, R. De Virgilio, Antonio Maccioni, M. Patrignani, and R. Torlone. Finding all maximal cliques in very large social networks. In EDBT, pages 173-184, 2016.

17 M. Cygan, F. V. Fomin, Ł. Kowalik, D. Lokshtanov, D. Marx, M. Pilipczuk, M. Pilipczuk, and S. Saurabh. Parameterized algorithms, volume 3. Springer, 2015.

18 N. Du, B. Wu, L. Xu, B. Wang, and X. Pei. A parallel algorithm for enumerating all maximal cliques in complex network. In Data Mining Workshops, 2006. ICDM Workshops 2006. Sixth IEEE International Conference on, pages 320-324. IEEE, 2006.

19 V. Dujmović, G. Fijavž, G. Joret, T. Sulanke, and D. R. Wood. On the maximum number of cliques in a graph embedded in a surface. European J. Combin., 32(8):1244-1252, 2011.

20 D. Eppstein, M. Löffler, and D. Strash. Listing All Maximal Cliques in Sparse Graphs in Near-Optimal Time, pages 403-414. Springer Berlin Heidelberg, Berlin, Heidelberg, 2010. doi : 10.1007/978-3-642-17517-6_36.

21 D. Eppstein and D. Strash. Listing all maximal cliques in large sparse real-world graphs. Experimental Algorithms, pages 364-375, 2011.

22 E. M. Eschen, C. T. Hoàng, J. P. Spinrad, and R. Sritharan. On graphs without a $C_{4}$ or a diamond. Discrete Appl. Math., 159(7):581-587, 2011.

23 M. Faloutsos, P. Faloutsos, and C. Faloutsos. On power-law relationships of the internet topology. In Proceedings of SIGCOMM, pages 251-262, 1999.

24 A. Ferrante, G. Pandurangan, and K. Park. On the hardness of optimization in power law graphs. In Proceedings of Conference on Computing and Combinatorics, pages 417-427, 2006.

25 A. Ferrante, G. Pandurangan, and K. Park. On the hardness of optimization in power-law graphs. Theoret. Comput. Sci., 393(1):220-230, 2008. doi:10.1016/j.tcs.2007.12.007.

26 S. Fortunato. Community detection in graphs. Physics Reports, 486:75-174, 2010.

27 J. Fox and F. Wei. On the number of cliques in graphs with a forbidden subdivision or immersion, 2016.

28 J. Fox and F. Wei. On the number of cliques in graphs with a forbidden minor. J. Combin. Theory Ser. B, 126:175-197, 2017. doi:10.1016/j.jctb.2017.04.004.

29 Jacob Fox, Tim Roughgarden, C. Seshadhri, Fan Wei, and Nicole Wein. Finding cliques in social networks: A new distribution-free model. arXiv preprint arXiv:1804.07431, 2018.

30 Z. Füredi and M. Simonovits. The History of Degenerate (Bipartite) Extremal Graph Problems, pages 169-264. Springer Berlin Heidelberg, Berlin, Heidelberg, 2013. doi: 10.1007/978-3-642-39286-3_7. 
31 L. Gąsieniec, M. Kowaluk, and A. Lingas. Faster multi-witnesses for boolean matrix multiplication. Information Processing Letters, 109(4):242-247, 2009.

32 M. Girvan and M. Newman. Community structure in social and biological networks. Proc. Natl. Acad. Sci. USA, 99(12):7821-7826, 2002. doi:10.1073/pnas.122653799.

33 R. Gupta, T. Roughgarden, and C. Seshadhri. Decompositions of triangle-dense graphs. SIAM J. Comput., 45(2):197-215, 2016.

34 M. Jha, C. Seshadhri, and A. Pinar. Path sampling: A fast and provable method for estimating 4-vertex subgraph counts. In World Wide Web $(W W W)$, pages 495-505, 2015.

35 J. M. Kleinberg. Navigation in a small world. Nature, 406:845, 2000.

36 J. M. Kleinberg. The small-world phenomenon: An algorithmic perspective. In Proceedings of the Symposium on Theory of Computing, pages 163-170, 2000.

37 J. M. Kleinberg. Small-world phenomena and the dynamics of information. In Advances in Neural Information Processing Systems, volume 1, pages 431-438, 2002.

38 R. Kumar, P. Raghavan, S. Rajagopalan, D. Sivakumar, A. Tomkins, and E. Upfal. Stochastic models for the web graph. In Proceedings of Foundations of Computer Science, pages 57-65, 2000. doi:10.1109/SFCS.2000.892065.

39 J. Leskovec, D. Chakrabarti, J. M. Kleinberg, C. Faloutsos, and Z. Ghahramani. Kronecker graphs: An approach to modeling networks. J. Mach. Learn. Res., 11:985-1042, 2010. URL: http://jmlr.csail.mit.edu/papers/v11/leskovec10a.html.

40 J. Leskovec, K. Lang, A. Dasgupta, and M. Mahoney. Community structure in large networks: Natural cluster sizes and the absence of large well-defined clusters. Internet Math., 6(1):29-123, 2008.

41 H. Lin, C. Amanatidis, M. Sideri, R. M. Karp, and C. H. Papadimitriou. Linked decompositions of networks and the power of choice in Polya urns. In Proceedings of the Symposium on Discrete Algorithms, pages 993-1002, 2008.

42 M. Mitzenmacher, J. Pachocki, R. Peng, C. Tsourakakis, and S. Xu. Scalable large nearclique detection in large-scale networks via sampling. In SIGKDD International Conference on Knowledge Discovery and Data Mining, pages 815-824, 2015. doi:10.1145/2783258. 2783385.

43 A. Montanari and A. Saberi. The spread of innovations in social networks. Proc. Natl. Acad. Sci. USA, 107(47):20196-20201, 2010.

44 J. Moon and L. Moser. On cliques in graphs. Israel J. Math., 3(1):23-28, 1965.

45 M. E. J. Newman. The structure of scientific collaboration networks. Proc. Natl. Acad. Sci. USA, 98(2):404-409, 2001. doi:10.1073/pnas.98.2.404.

46 M. E. J. Newman. Properties of highly clustered networks. Physical Review E, 68(2):026121, 2003. doi:10.1103/PhysRevE.68.026121.

47 M. E. J. Newman. Finding community structure in networks using the eigenvectors of matrices. Phys. Rev. E, 74(3):036104, 2006. doi:10.1103/PhysRevE.74.036104.

48 V. Nikiforov. The number of cliques in graphs of given order and size. Trans. Amer. Math. Soc., 363(3):1599-1618, 2011.

49 V. Raman and S. Saket. Short cycles make $W$-hard problems hard: FPT algorithms for $W$-hard problems in graphs with no short cycles. Algorithmica, 52(2):203-225, 2008.

50 A. A. Razborov. On the minimal density of triangles in graphs. Comb. Probab. Comput., 17(4):603-618, 2008. doi:10.1017/S0963548308009085.

51 C. Reiher. The clique density theorem. Ann. of Math. (2), 184(3):683-707, 2016. doi: 10.4007/annals. 2016.184.3.1.

52 R. A. Rossi, D. F. Gleich, and A. H. Gebremedhin. Parallel maximum clique algorithms with applications to network analysis. SIAM J. Sci. Comput., 37(5), 2015. doi:10.1137/ 14100018X. 
53 A. Sala, L. Cao, C. Wilson, R. Zablit, H. Zheng, and B. Y. Zhao. Measurement-calibrated graph models for social network experiments. In Proceedings of the World Wide Web Conference, pages 861-870. ACM, 2010. doi:10.1145/1772690.1772778.

54 A. E. Sarıüce, C. Seshadhri, A. Pınar, and Ü. V. Çatalyürek. Finding the hierarchy of dense subgraphs using nucleus decompositions. In Proceedings of the 24th International Conference on World Wide Web, WWW '15, pages 927-937, Republic and Canton of Geneva, Switzerland, 2015. International World Wide Web Conferences Steering Committee. URL: http://dl .acm.org/citation. cfm?id=2736277.2741640.

55 D. Saxton and A. Thomason. Hypergraph containers. Invent. Math., 201(3):925-992, Sep 2015. doi:10.1007/s00222-014-0562-8.

56 C. Seshadhri, A. Pinar, and T. G. Kolda. Fast triangle counting through wedge sampling. In Proceedings of the SIAM Conference on Data Mining, 2013. URL: http://arxiv.org/ abs/1202.5230.

57 E. Tomita and T. Kameda. An efficient branch-and-bound algorithm for finding a maximum clique with computational experiments. J. Global Optim., 37(1):95-111, 2007.

58 E. Tomita, A. Tanaka, and H. Takahashi. The worst-case time complexity for generating all maximal cliques and computational experiments. Theor. Comput. Sci., 363(1):28-42, 2006. doi:10.1016/j.tcs.2006.06.015.

59 C. Tsourakakis. The k-clique densest subgraph problem. In Proceedings of the 24th International Conference on World Wide Web, WWW '15, pages 1122-1132, Republic and Canton of Geneva, Switzerland, 2015. International World Wide Web Conferences Steering Committee. URL: http://dl . acm.org/citation. cfm?id=2736277 . 2741098.

60 C. Tsourakakis, F. Bonchi, A. Gionis, F. Gullo, and M. Tsiarli. Denser than the densest subgraph: Extracting optimal quasi-cliques with quality guarantees. In Proc. of the 19th ACM SIGKDD International Conference on Knowledge Discovery and Data Mining, KDD '13, 2013.

61 S. Tsukiyama, M. Ide, H. Ariyoshi, and I. Shirakawa. A new algorithm for generating all the maximal independent sets. SIAM J. Comput., 6(3):505-517, 1977.

62 J. Ugander, L. Backstrom, and J. Kleinberg. Subgraph frequencies: Mapping the empirical and extremal geography of large graph collections. In Proceedings of World Wide Web Conference, pages 1307-1318, 2013.

63 J. Ugander, B. Karrer, L. Backstrom, and C. Marlow. The anatomy of the facebook social graph. arXiv preprint arXiv:1111.4503, 2011.

64 D. Watts and S. Strogatz. Collective dynamics of 'small-world' networks. Nature, 393:440442, 1998. doi:10.1038/30918. 